\title{
BENDING STIFFNESS OF A MULTILAYERED PLATE
}

\author{
Petr E. Tovstik ${ }^{1}$, Tatiana M. Tovstik ${ }^{2}$ \\ ${ }^{1,2}$ Sankt-Petersburg State University, Universitetskaya nab. 7/9, Sankt-Petersburg, Russian Federation \\ e-mail: peter.tovstik@mail.ru
}

Keywords: Multilayered Plate, Bending Stiffness, Asymptotic Methods, Monte Carlo Method.

\begin{abstract}
A thin elastic multilayered plate consisting of alternating hard and soft isotropic layers is studied. One of the face planes is subject to a normal pressure and the other face plane is free. A formula for the deflection of an infinite plate under a doubly periodic external force is delivered using asymptotic expansions. This formula is also applied for a rectangular plate with Navier boundary conditions on its edges. The maximal deflection is accepted as a measure of the plate stiffness. The purpose of the present paper is to obtain an expression for the plate deflection and find an optimal distribution of hard and soft layers assuming that their total thicknesses are given. The Monte Carlo method is used for finding the optimal distribution of layers.
\end{abstract}




\section{INTRODUCTION}

The 2D classical equation for a plate was obtained by Sophie Germain in 1808 with the purpose of explaining Chladni figures. The equation of a plate bending can be obtained on the basis of the Kirchhoff-Love (KL) hypotheses [1,2]. The more involved and sometimes more exact equation, which takes into account the transversal shear, follows from the TimoshenkoReissner (TR) hypotheses [3, 4].

The 2D models of plates and shells are chiefly based on the 3D equations of the theory of elasticity. The methods of unknown functions expansions in series of Legendre polynomials in the thickness direction were used in [5, 6]. Numerous investigations [7-9] were devoted to the derivation of $2 \mathrm{D}$ equations by using asymptotic expansions in power series in the small parameter $\mu=h / L$, which is equal to the dimensionless plate thickness ( $h$ and $L$ are, respectively, the thickness and the typical wave length in the tangential directions). A different approach $[10,11]$ rests on the direct derivation of $2 \mathrm{D}$ equations of plates and shells without referring to a 3D media. An account of general problems of the plates theory may be found in the books [12-14].

The present paper is concerned with a thin plate of constant thickness made of a linearly elastic material that is transversally isotropic and heterogeneous in the thickness direction. For a transversally isotropic material the accepted simplification [15], for which a 3D system of sixth order of the theory of elasticity splits into systems of second and fourth orders, is possible. Asymptotic expansions in powers of the small thickness parameter $\mu$ are constructed, the bending equation of second-order accuracy (the SA model) is obtained. The origin of this paper is the paper [16], in which an isotropic homogeneous plate is studied, and the paper [8], in which a heterogeneous plate is briefly examined. For a multi-layer plate with alternating hard and soft layers, an explicit formula based on the SA model for the maximum deflection is delivered. The results of the SA model are compared with the KL classical model and also with the exact numerical solution. The ratio $\eta=E_{2} / E_{1}$ of the soft and hard Young moduli changes in a very wide range $(0.0001 \leq \eta \leq 1)$.

The aforementioned formula is obtained for an infinite in the tangential directions plate subject to a doubly periodic external load. After employing the Fourier expansions this formula is applied to a rectangular plate with the Navier boundary conditions.

For a multi-layer plate with alternating hard and soft layers, the distribution of layer thicknesses, which gives the maximum bending stiffness, is found. Here it is assumed that the summary thicknesses of the hard and of the soft layers are given. This problem is attacked by the Monte Carlo method. For a very small ratio $\eta$ of elastic moduli, the three-layer plate with the soft layer lying between two hard layers was found not to be optimal. In this case, the plate with the maximum bending stiffness is multi-layered one with the number of layers $n>3$.

\section{EQUILIBRIUM EQUATIONS AND THEIR SIMPLIFICATION}

Consider the linear bending problem of a thin plate made of a transversally isotropic heterogeneous material. The $3 \mathrm{D}$ equilibrium equations are

$$
\frac{\partial \sigma_{i j}}{\partial x_{j}}+f_{i}=0, \quad i, j=1,2,3, \quad 0 \leq x_{3}=z \leq h,
$$

where $x_{j}$ are the Cartesian coordinates, $f_{i}$ are the projections of the external load intensity, the summation being carried out over repeating subscripts. 
The stresses $\sigma_{i j}$ are related to the strains $\varepsilon_{i j}$ as follows:

$$
\begin{array}{rlrl}
\sigma_{11} & =E_{11} \varepsilon_{11}+E_{12} \varepsilon_{22}+E_{13} \varepsilon_{33}, & & \sigma_{12}=G_{12} \varepsilon_{12}, \\
\sigma_{22}=E_{12} \varepsilon_{11}+E_{11} \varepsilon_{22}+E_{13} \varepsilon_{33}, & & \sigma_{13}=G_{13} \varepsilon_{13}, \\
\sigma_{33}=E_{13} \varepsilon_{11}+E_{13} \varepsilon_{22}+E_{33} \varepsilon_{33}, & & \sigma_{23}=G_{13} \varepsilon_{23}, \\
\varepsilon_{11}=\frac{\partial u_{1}}{\partial x_{1}}, \quad \varepsilon_{12}=\frac{\partial u_{1}}{\partial x_{2}}+\frac{\partial u_{2}}{\partial x_{1}}, & \text { etc. } &
\end{array}
$$

Here, $E_{11}=E_{12}+2 G_{12}, u_{i}$ are deflections. The elastic moduli $E_{i j}, G_{i j}$ are independent of the tangential coordinates $x_{1}, x_{2}$, but they may depend on the transversal coordinate $x_{3}=z$. For functionally gradient materials the moduli are continuous functions in $z$, and for multi-layered plates they are piecewise continuous functions.

For an isotropic material

$$
E_{11}=E_{33}=\frac{E(1-\nu)}{(1+\nu)(1-2 \nu)}, \quad E_{12}=E_{13}=\frac{E \nu}{(1+\nu)(1-2 \nu)}, \quad G_{12}=G_{13}=G=\frac{E}{2(1+\nu)},
$$

where $E$ and $\nu$ are the Young's modulus and the Poisson ratio, respectively.

We set the homogeneous boundary conditions on the face planes $z=0$ and $z=h$

$$
\sigma_{i 3}=0, \quad i=1,2,3 .
$$

If the surface forces are given, then they are included in the body forces by using the Dirac delta-function.

Introduce new unknown functions $u, v, \sigma, \tau$ as

$$
\begin{aligned}
u & =\frac{\partial u_{1}}{\partial x_{1}}+\frac{\partial u_{2}}{\partial x_{2}}, & v & =\frac{\partial u_{1}}{\partial x_{2}}-\frac{\partial u_{2}}{\partial x_{1}}, \\
\sigma & =\frac{\partial \sigma_{13}}{\partial x_{1}}+\frac{\partial \sigma_{23}}{\partial x_{2}}, & \tau & =\frac{\partial \sigma_{13}}{\partial x_{2}}-\frac{\partial \sigma_{23}}{\partial x_{1}} .
\end{aligned}
$$

For a transversally isotropic material the system (1), (2) is split into two subsystems [15]:

$$
\begin{aligned}
& \frac{\partial \tau}{\partial z}+G_{12} \Delta v+m_{1}=0, \quad \tau=G_{13} \frac{\partial v}{\partial z}, \quad \Delta=\frac{\partial^{2}}{\partial x_{1}^{2}}+\frac{\partial^{2}}{\partial x_{2}^{2}}, \quad m_{1}=\frac{\partial f_{1}}{\partial x_{2}}-\frac{\partial f_{2}}{\partial x_{1}} \\
& \sigma_{33}=E_{13} u+E_{33} \frac{\partial w}{\partial z}, \quad \sigma=G_{13}\left(\frac{\partial u}{\partial z}+\Delta w\right), \quad w=u_{3}, \\
& \frac{\partial \sigma}{\partial z}+E_{0} \Delta u+\frac{E_{13}}{E_{33}} \Delta \sigma_{33}+m=0, \quad \frac{\partial \sigma_{33}}{\partial z}+\sigma+f_{3}=0, \quad m=\frac{\partial f_{1}}{\partial x_{1}}+\frac{\partial f_{2}}{\partial x_{2}},
\end{aligned}
$$

with

$$
E_{0}=E_{11}-\frac{E_{13}^{2}}{E_{33}}=\frac{E}{1-\nu^{2}} .
$$

System (6) of the second differential order in $z$ describes the boundary layer and will not be studied here.

The fourth order system (7) describes the plate bending. The 2D plate model is obtained here by using asymptotic expansions in powers of the small parameter $\mu=h / L[8,17]$. 
We introduce the dimensionless variables (denoted with hats)

$$
\begin{aligned}
& \left\{u_{1}, u_{2}, w, z\right\}=h\left\{\hat{u}_{1}, \hat{u}_{2}, \hat{w}, \hat{z}\right\}, \quad\left\{x_{1}, x_{2}\right\}=L\left\{\hat{x}_{1}, \hat{x}_{2}\right\}, \quad u=\mu \hat{u}, \\
& \left\{\sigma_{i j}, \sigma, E_{i j}, G_{i j}, E_{0}\right\}=E_{*}\left\{\hat{\sigma}_{i j}, \hat{\sigma}, \hat{E}_{i j}, \hat{G}_{i j}, c_{0}\right\}, \quad f_{i}=\frac{E_{*}}{h} \hat{f}_{i}, \quad E_{*}=\frac{1}{h} \int_{0}^{h} E_{0}(z) d z,
\end{aligned}
$$

and rewrite system (7) in the dimensionless form

$$
\begin{array}{ll}
\frac{\partial \hat{w}}{\partial \hat{z}}=-\mu c_{\nu} \hat{u}+c_{3} \hat{\sigma}_{33}, & \frac{\partial \hat{u}}{\partial \hat{z}}=-\mu \hat{\Delta} \hat{w}+c_{g} \hat{\sigma}, \\
\frac{\partial \hat{\sigma}}{\partial \hat{z}}=Y_{3}(\hat{z})=-\mu^{2} c_{0} \hat{\Delta} \hat{u}-\mu c_{\nu} \hat{\Delta} \hat{\sigma}_{33}-\hat{m}, & \frac{\partial \hat{\sigma}_{33}}{\partial \hat{z}}=Y_{4}(\hat{z})=-\mu \hat{\sigma}-\hat{f}_{3}, \quad 0 \leq \hat{z} \leq 1,
\end{array}
$$

with

$$
\begin{aligned}
& c_{\nu}=\frac{E_{13}}{E_{33}}=\frac{\nu}{1-\nu}, \quad c_{3}=\frac{E_{*}}{E_{33}}, \quad c_{g}=\frac{E_{*}}{G_{13}}, \\
& \hat{\sigma}=\frac{\partial \hat{\sigma}_{13}}{\partial \hat{x}_{1}}+\frac{\partial \hat{\sigma}_{23}}{\partial \hat{x}_{2}}, \quad \hat{m}=\frac{\partial \hat{f}_{1}}{\partial \hat{x}_{1}}+\frac{\partial \hat{f}_{2}}{\partial \hat{x}_{2}}, \quad \hat{\Delta}=\frac{\partial^{2}}{\partial \hat{x}_{1}^{2}}+\frac{\partial^{2}}{\partial \hat{x}_{2}^{2}} .
\end{aligned}
$$

Here, $L$ is the typical value of the wave length in the tangential directions, and $E_{*}$ is the average value of the modulus $E_{0}$. The dimensionless coefficients $c_{0}, c_{\nu}, c_{g}, c_{3}$ are the given functions of $\hat{z}$. From the boundary conditions (4) we get

$$
\hat{\sigma}=\hat{\sigma}_{33}=0 \quad \text { at } \quad \hat{z}=0 \quad \text { and } \quad \hat{z}=1 .
$$

In what follows, the hat sign will be omitted.

\section{ASYMPTOTIC SOLUTION OF THE BOUNDARY VALUE PROBLEM (9), (11)}

Assume that the dimensionless external forces $f_{3}, m$ are of the order of unity. Then the orders of the unknown functions are

$$
\sigma_{33}=O(1), \quad \sigma=O\left(\mu^{-1}\right), \quad u=O\left(\mu^{-3}\right), \quad w=O\left(\mu^{-4}\right) .
$$

The right-hand sides of Eqs. (9) are small, and the method of iterations $[10,18]$ is used. To construct the solution of second-order accuracy we seek it as

$$
w=\mu^{-4} w_{0}+\mu^{-2} w_{2}, \quad u=\mu^{-3} u_{0}+\mu^{-1} u_{2}, \quad \sigma=\mu^{-1} \sigma_{0}+\mu \sigma_{2}, \quad \sigma_{33}=\sigma_{33,0}+\mu^{2} \sigma_{33,2} .
$$

The arbitrary functions $w^{0}\left(x_{1}, x_{2}\right)$ and $u^{0}\left(x_{1}, x_{2}\right)$ appear after the integration in $z$ of the first two equations (9). These functions are found from the compatibility conditions of the remaining two equations (9) and the boundary conditions (11) [17]

$$
\left\langle Y_{3}(z)\right\rangle=0, \quad\left\langle Y_{4}(z)\right\rangle=0, \quad\langle Z(z)\rangle \equiv \int_{0}^{1} Z(z) d z .
$$

In the zero approximation we get

$$
\begin{aligned}
& w_{0}=w_{0}\left(x_{1}, x_{2}\right), \quad u_{0}=(a-z) \Delta^{2} w_{0}, \quad a=\left\langle z c_{0}(z)\right\rangle, \\
& \sigma_{0}=\varphi_{1}(z) \Delta^{2} w_{0}^{0}, \quad \varphi_{1}(z)=\int_{0}^{z} c_{0}(z)(z-a) d z, \\
& D \Delta^{2} w_{0}=F_{3}, \quad D=\left\langle(z-a)^{2} c_{0}(z)\right\rangle, \quad F_{3}=\left\langle f_{3}(z)\right\rangle, \\
& \sigma_{33,0}=-\frac{F_{3}}{D} \varphi_{2}-\varphi_{3}, \quad \varphi_{2}(z)=\int_{0}^{z} \varphi_{1}(z) d z, \quad \varphi_{3}\left(x_{1}, x_{2}, z\right)=\int_{0}^{z} f_{3}\left(x_{1}, x_{2}, z\right) d z,
\end{aligned}
$$


where $z=a$ is the position of the plate neutral layer, $D$ is the bending stiffness of a plate with the variable elastic moduli, $F_{3}$ is the full transversal force. The equation $D \Delta^{2} w^{0}=F_{3}$ corresponds to the classical KL model.

In the second approximation the solution is more unwieldy. Here, we give only the function $w_{2}$, which depends on $z$. At $z=0$ it satisfies the equation

$$
D \Delta^{2} w_{2}(0)=A \Delta F_{3}+N\left(\Delta f_{3}\right)-M, \quad A=A_{g}-A_{\nu}
$$

where

$$
\begin{aligned}
& N\left(\Delta f_{3}\right)=\int_{0}^{1} c_{\nu}(z)(a-z)\left(\int_{0}^{z} \Delta f_{3} d z_{1}\right) d z, \quad M=\int_{0}^{1}(a-z) m(z) d z \\
& A_{g}=\frac{1}{D} \int_{0}^{1} c_{0}(z)(z-a) \int_{0}^{z} c_{g}\left(z_{1}\right) \int_{0}^{z_{1}} c_{0}\left(z_{2}\right)\left(z_{2}-a\right) d z_{2} d z_{1} d z \\
& A_{\nu}=\frac{1}{D} \int_{0}^{1}(z-a) \int_{0}^{z} \int_{0}^{z_{1}}\left(c_{\nu}(z) c_{0}\left(z_{2}\right)+c_{0}(z) c_{\nu}\left(z_{2}\right)\right)\left(z_{2}-a\right) d z_{2} d z_{1} d z
\end{aligned}
$$

The full deflection of the reference plane $z=0$ satisfies the equation

$$
D \mu^{4} \Delta^{2} w(0)=F_{3}+\mu^{2}\left(A \Delta F_{3}+L\left(\Delta f_{3}\right)-M\right)+O\left(\mu^{4}\right),
$$

in which the coefficients $D$ and $A$ depend of the elastic moduli distribution in the plate thickness, the summands $L\left(\Delta f_{3}\right)$ and $M$ depend on the distribution of external transversal and tangential loads, respectively. The summands $A_{g}$ and $A_{\nu}$ take into account the transversal shear and the Poisson's strains, respectively.

The full deflection $w(z)$ of the arbitrary plane $z$ is expressed through $w(0)$ as

$$
w(z)=w(0)+\mu^{2} \Delta w(0) \int_{0}^{z} c_{\nu}(z)(z-a) d z .
$$

\section{MULTILAYERED PLATE UNDER A NORMAL PRESSURE}

Consider a multi-layered plate with hard and soft isotropic homogeneous layers under a normal pressure $F_{3}$ acting on the plane $z=0$. In this case, in Eq. (18) $L\left(\Delta f_{3}\right)=\left\langle c_{\nu}(a-z)\right\rangle \Delta F_{3}$ and $M=0$, and so Eq. (18) assumes the form

$$
D \mu^{4} \Delta^{2} w(0)=F_{3}+\mu^{2} A_{1} \Delta F_{3}, \quad A_{1}=A+\int_{0}^{1} c_{\nu}(z)(z-a) d z .
$$

If the plate is homogeneous one-layered, then the elastic moduli $c_{0}, c_{g}, c_{\nu}$ are constant, and so the integrals in (17) can be taken, and so in Eq. (20) we have

$$
D \mu^{4} \Delta^{2} w(0)=F_{3}+\mu^{2} A_{1} \Delta F_{3}, \quad A_{1}=\frac{2 c_{\nu}-c_{g}}{10}=-\frac{1}{5}, \quad D=\frac{1}{12} .
$$

Using the neutral plane $z=1 / 2$ as a reference one due to Eq. (19) we get

$$
D \mu^{4} \Delta^{2} w(1 / 2)=F_{3}+\mu^{2} A_{2} \Delta F_{3}, \quad A_{2}=\frac{3 c_{\nu}-4 c_{g}}{40}=\frac{3 \nu-8}{40(1-\nu)} .
$$

This value $A_{2}$ was obtained in [16] and was repeated in [8]. 
Consider a plate of thickness $h$ consisting of $n=2 n_{0}+1$ homogeneous isotropic layers of thickness $h_{k}, k=1,2, \ldots, n\left(h=\sum h_{k}\right)$. Let $E_{1}, \nu_{1}$ and $E_{2}, \nu_{2}\left(E_{2} / E_{1} \leq 1\right)$ be the Young's moduli and the Poisson ratios of hard layers with odd numbers and of soft layers with even numbers, respectively. We set

$$
z_{0}=0, \quad z_{k}=\sum_{i=1}^{k} h_{i}, \quad k=1, \ldots, n .
$$

The elastic moduli are known to be piecewise functions in $z$, and so we put

$$
\begin{array}{rlrl}
e_{k} & =\frac{E_{1}}{1-\nu_{1}^{2}}, \quad c_{k}=\frac{\nu_{1}}{1-\nu_{1}}, & g_{k}=\frac{E_{1}}{2\left(1+\nu_{1}\right)}, & k \text { is odd }, \\
e_{k}=\frac{E_{2}}{1-\nu_{2}^{2}}, & c_{k}=\frac{\nu_{2}}{1-\nu_{2}}, \quad g_{k}=\frac{E_{2}}{2\left(1+\nu_{2}\right)}, & k \text { is even } .
\end{array}
$$

Calculating the integrals in Eqs. (15) and (17) we find the coordinate $z=a$ of the neutral layer, the bending stiffness $D$ according to the KL model, and the coefficients $A_{g}$ and $A_{\nu}$ in Eqs. (17) as follows:

$$
\begin{aligned}
& a=\frac{1}{2} \sum_{k=1}^{n} e_{k}\left(z_{k}^{2}-z_{k-1}^{2}\right)\left(\sum_{k=1}^{n} e_{k} h_{k}\right)^{-1}, \quad D=\frac{1}{3} \sum_{k=1}^{n} e_{k}\left(\hat{z}_{k}^{3}-\hat{z}_{k-1}^{3}\right), \quad \hat{z}_{k}=z_{k}-a, \\
& A_{g}=\frac{1}{D} \sum_{k=1}^{n}\left(\frac{e_{k} f_{1 k}}{2}\left(\hat{z}_{k}^{2}-\hat{z}_{k-1}^{2}\right)+\frac{f_{2 k}}{3 g_{k}}\left(\hat{z}_{k}^{3}-\hat{z}_{k-1}^{3}\right)+\frac{e_{k}}{30 g_{k}}\left(\hat{z}_{k}^{5}-\hat{z}_{k-1}^{5}\right)\right), \\
& A_{\nu}=\frac{1}{D} \sum_{k=1}^{n}\left(\frac{e_{k} f_{3 k}^{c}+c_{k} f_{3 k}^{e}}{2}\left(\hat{z}_{k}^{2}-\hat{z}_{k-1}^{2}\right)+\left(c_{k} f_{4 k}^{e}+e_{k} f_{4 k}^{c}\right)\left(\hat{z}_{k}^{3}-\hat{z}_{k-1}^{3}\right)+\frac{e_{k} c_{k}}{15}\left(\hat{z}_{k}^{5}-\hat{z}_{k-1}^{5}\right)\right),
\end{aligned}
$$

where

$$
\begin{aligned}
f_{1 k} & =\sum_{i=1}^{k-1}\left(\frac{f_{2 i} h_{i}}{g_{i}}+\frac{e_{i}}{6 g_{i}}\left(\hat{z}_{i}^{3}-\hat{z}_{i-1}^{3}\right)\right)-\frac{f_{2 k} h_{k}}{g_{k}}-\frac{e_{k}}{6 g_{k}} \hat{z}_{k}^{3}, \\
f_{2 k} & =\frac{1}{2} \sum_{i=1}^{k-1} e_{i}\left(\hat{z}_{i}^{2}-\hat{z}_{i-1}^{2}\right)-\frac{1}{2} e_{k} \hat{z}_{k}^{2}, \\
f_{3 k}^{e} & =\sum_{i=1}^{k-1}\left(f_{4 i}^{e} h_{i}+\frac{e_{i}}{6}\left(\hat{z}_{i}^{3}-\hat{z}_{i-1}^{3}\right)\right)-f_{4 k}^{e} h_{k}-\frac{e_{k}}{6} \hat{z}_{k}^{3}, \\
f_{4 k}^{e} & =\frac{1}{2} \sum_{i=1}^{k-1} e_{i}\left(\hat{z}_{i}^{2}-\hat{z}_{i-1}^{2}\right)-\frac{1}{2} e_{k} \hat{z}_{k}^{2}, \quad(e \rightarrow c) .
\end{aligned}
$$

Here, $(e \rightarrow c)$ means that the similar formulae also hold for the moduli $c_{k}$.

Assume, at first, that the plate is infinite $\left(-\infty<x_{1}, x_{2}<\infty\right)$, and consider a doubly periodic normal pressure $F_{3}=F_{3}^{0} \sin r_{1} x_{1} \sin r_{2} x_{2}$. Then the deflection $W\left(x_{1}, x_{2}, z\right)=$ $W(z) \sin r_{1} x_{1} \sin r_{2} x_{2}$ is also doubly periodic. We rewrite Eq. (20) as

$$
D r^{4} W(0)=\left(1-r^{2} A_{1}\right) F_{3}^{0},
$$

where

$$
A_{1}=A_{g}-A_{\nu}-\frac{1}{2} \sum_{k=1}^{n} c_{k}\left(\hat{z}_{k}^{2}-\hat{z}_{k-1}^{2}\right), \quad r^{2}=r_{1}^{2}+r_{2}^{2}
$$


and the coefficients $D, A_{\nu}, A_{g}$ are given in Eqs. (25).

In the dimensionless form (with hats)

$$
W(0)=h w(0), \quad D=h^{3} \hat{D}, \quad A_{1}=h^{2} \hat{A}_{1}, \quad \mu=r h
$$

Equation (27) gives the deflection of the second-order accuracy (SA)

$$
w(0)^{S A}=w^{K L}\left(1-\mu^{2} \hat{A}_{1}\right), \quad w^{K L}=\frac{F_{3}^{0} h}{\mu^{4} \hat{D}},
$$

where $w^{K L}$ is the deflection in the KL model.

A comparison of the expressions $\mu=r h$ and $\mu=h / L$ leads to the conclusion that in this problem the typical wave length $L$ should be taken as

$$
L=\frac{1}{r}=\frac{1}{\sqrt{r_{1}^{2}+r_{2}^{2}}} .
$$

To discuss the errors of the approximate expressions (30) for $w^{K L}$ and $w(0)^{S A}$ we require some numerical examples. We consider a multi-layered plate with $n=11$ layers and $h=1$, $\nu_{1}=\nu_{2}=0.3, F_{3}^{0}=1$, and take $h_{k}=1 / 18, E_{k}=1, k=2 i-1, \mathrm{i}=1, \ldots, 6$, for the hard layers, and $h_{k}=2 / 15, E_{k}=\eta, k=2 i, i=1, \ldots, 5$, for the soft layers. Such a plate will be referred to as a plate with uniform thickness distribution. We take two values of the relative thickness parameter: $\mu=0.3776$ and $\mu=0.2221$. The ratio $\eta=E_{2} / E_{1}$ of the elastic moduli will vary in the range $0.0001 \leq \eta \leq 1$. We compare the approximate values (30) with the exact value $w^{e}$ found by numerical solution of Eqs. (9). The results are given in Table 1. Columns 2-4 correspond to $\mu=0.3776$, and columns 5-7, to $\mu=0.2221$.

\begin{tabular}{|l||r|rc||r|rc|}
\hline \multicolumn{1}{|c||}{1} & \multicolumn{1}{c|}{2} & \multicolumn{1}{c|}{3} & \multicolumn{1}{c|}{5} & \multicolumn{1}{c|}{5} & \multicolumn{1}{c|}{6} & \multicolumn{1}{c|}{7} \\
\hline \multicolumn{1}{|c||}{$\eta$} & $w^{e}$ & $w^{K L} / w^{e}$ & $w^{S A} / w^{e}$ & $w^{e}$ & $w^{K L} / w^{e}$ & $w^{S A} / w^{e}$ \\
\hline 1.0 & 553 & 0.9717 & 0.9994 & 4529 & 0.9902 & 0.9999 \\
0.31 & 936 & 0.9539 & 0.9992 & 7578 & 0.9837 & 0.9999 \\
0.1 & 1270 & 0.8899 & 0.9985 & 9830 & 0.9532 & 0.9998 \\
0.031 & 1684 & 0.7322 & 0.9975 & 11587 & 0.8882 & 0.9996 \\
0.01 & 2700 & 0.4703 & 0.9987 & 14723 & 0.7198 & 0.9998 \\
0.0031 & 5746 & 0.2231 & 1.0110 & 23692 & 0.4510 & 1.0036 \\
0.001 & 14258 & 0.0800 & 1.0441 & 51150 & 0.2098 & 1.0192 \\
0.00031 & 41793 & 0.0308 & 1.1156 & 134232 & 0.0800 & 1.0557 \\
0.0001 & 110822 & 0.0116 & 1.3054 & 374835 & 0.0287 & 1.1337 \\
\hline
\end{tabular}

Table 1: Error of the approximate models for some values of $\eta$ with $\mu=0.3776$ and $\mu=0.2221$.

The relative error of the KL and SA models is found by comparing the results in columns $3,4,6,7$ with one. The exactness of the KL model is of first asymptotical order with respect to $\mu$, and it is acceptable for applications only for $0.1 \leq \eta \leq 1$. The SA model of second asymptotical order may be used in the very wide range $0.001 \leq \eta \leq 1$. For $0.1 \leq \eta \leq 1$ the SA model is essentially more exact than the TR model. For $0.0001 \leq \eta \leq 0.001$ the $2 \mathrm{D}$ models are unacceptable.

The errors of the 2D models decrease simultaneously with the thickness parameter $\mu$. This follows from the comparison of columns 3 and 6, and also of columns 4 and 7 . 


\section{DEFLECTION OF A RECTANGULAR MULTILAYERED PLATE}

Consider a rectangular multi-layer plate with $0 \leq x_{1} \leq a_{1}, 0 \leq x_{2} \leq a_{2}, 0 \leq z \leq h$ (Fig. 1). In the previous sections the boundary conditions at the plate edges were either not imposed or a plate was assumed to be infinite in the tangential directions. Now the following variant of boundary conditions will be accepted:

$$
\begin{aligned}
& u_{2}=w=\sigma_{11}=0 \quad \text { at } \quad x_{1}=0, \quad x_{1}=a_{1}, \\
& u_{1}=w=\sigma_{22}=0 \quad \text { at } \quad x_{2}=0, \quad x_{2}=a_{2},
\end{aligned}
$$

(the so-called Navier conditions) and

$$
\sigma_{13}=\sigma_{23}=0, \sigma_{33}=-F_{3}^{0} \quad \text { at } \quad z=0 ; \quad \sigma_{13}=\sigma_{23}=\sigma_{33}=0 \quad \text { at } \quad z=h .
$$

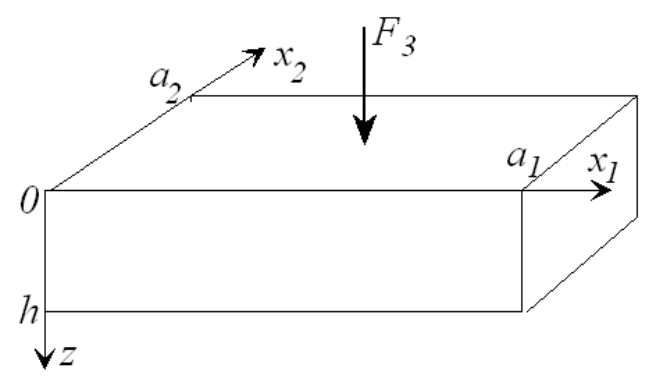

Fig. 1. A plate.

The functions

$$
\begin{aligned}
& u_{1}\left(x_{1}, x_{2}, z\right)=r_{1 m} u(z) \cos \left(r_{1 m} x_{1}\right) \sin \left(r_{2 n} x_{2}\right), \\
& u_{2}\left(x_{1}, x_{2}, z\right)=r_{2 n} u(z) \sin \left(r_{1 m} x_{1}\right) \cos \left(r_{2 n} x_{2}\right), \quad r_{1 m}=\frac{m \pi}{a_{1}}, r_{2 n}=\frac{n \pi}{a_{2}}, \quad m, n=1,2, \ldots, \\
& w\left(x_{1}, x_{2}, z\right)=w(z) \sin \left(r_{1 m} x_{1}\right) \sin \left(r_{2 n} x_{2}\right),
\end{aligned}
$$

satisfy the boundary conditions (32). The boundary layer does not appear, because $v=\partial u_{2} / \partial x_{1}-\partial u_{1} / \partial x_{2} \equiv 0$ (see Eqs. (5)).

Eq. (30) for the deflection is delivered for a doubly periodic external force $F_{3}=F_{3}^{0} \sin \left(r_{1} x_{1}\right) \sin \left(r_{2} x_{2}\right)$ with $\mu=h \sqrt{r_{1}^{2}+r_{2}^{2}}$. To use Eq. (30) for a constant force $F_{3}$ in the rectangle $0 \leq x_{1} \leq a_{1}, 0 \leq x_{2} \leq a_{2}$ we expand it in a double Fourier series

$$
F_{3}=F_{3} \sum_{m, n=1,3, \ldots} \frac{16}{m n \pi^{2}} \sin \left(r_{1 m} x_{1}\right) \sin \left(r_{2 n} x_{2}\right),
$$

and apply Eq. (30) to the each summand of (35). In this case

$$
\mu=\mu_{m n}=h \sqrt{\left(\frac{m \pi}{a_{1}}\right)^{2}+\left(\frac{n \pi}{a_{2}}\right)^{2}}, \quad m, n=1,2, \ldots
$$

and so the full deflection is

$$
w\left(x_{1}, x_{2}, 0\right)=\frac{F_{3}}{D} \sum_{m, n=1,3, \ldots} C_{m n} \sin \left(r_{1 m} x_{1}\right) \sin \left(r_{2 n} x_{2}\right), \quad C_{m n}=\frac{16\left(1-\mu_{m n}^{2} A_{1}\right)}{m n \pi^{2} \mu_{m n}^{4}},
$$


where $D$ and $A_{1}$ are given in (25) and (28), respectively. The similar expression is valid for the deflection of the opposite face plane $w\left(x_{1}, x_{2}, h\right)$. In this case, the constant $A_{1}$ in Eq. (37) needs to be replaced by $A=A_{g}-A_{\nu}$.

The deflection of the line $x_{2}=a_{2} / 2, z=0$ and the maximum deflection at $z=0$ are, respectively,

$$
\begin{aligned}
& w\left(x_{1}, a_{2} / 2,0\right)=\frac{F_{3}}{D} \sum_{k=0}^{\infty} B_{2 k+1} \sin \left(r_{1,2 k+1} x_{1}\right), \quad B_{2 k+1}=\sum_{j=0}^{\infty}(-1)^{j} C_{2 k+1,2 j+1}, \\
& w\left(a_{1} / 2, a_{2} / 2,0\right)=\frac{F_{3}}{D} A_{0}, \quad A_{0}=\sum_{k, j=0}^{\infty}(-1)^{k+j} C_{2 k+1,2 j+1}=\sum_{k=0}^{\infty}(-1)^{k} B_{2 k+1} .
\end{aligned}
$$

All the series in (37) and (38) converge rapidly, the summands with $C_{11}$ are much larger than the remaining summands (see Table 4).

As an example, we consider a multi-layer square plate with $n=11$ and with the same data as in Section 4. We assume in addition that $a_{1}=a_{2}=20$, and so $\mu=\mu_{11}=0.2221$. For various values of $\eta=E_{2} / E_{1}$, the first coefficients $C_{2 k+1,2 j+1}, B_{2 k+1}$, and $A_{0}$ are given in Table 2 .

\begin{tabular}{|r|cccccc|ccc|c|}
\hline$\eta$ & $C_{11}$ & $C_{13}$ & $C_{33}$ & $C_{15}$ & $C_{35}$ & $C_{55}$ & $B_{1}$ & $B_{3}$ & $B_{5}$ & $A_{0}$ \\
\hline 1 & 4528 & 191 & 62 & 31 & 19 & 10 & 4368 & 160 & 22 & 4230 \\
0.1 & 9828 & 463 & 164 & 89 & 58 & 32 & 9454 & 388 & 63 & 9129 \\
0.01 & 14720 & 1255 & 593 & 383 & 282 & 183 & 13848 & 1045 & 284 & 13087 \\
0.001 & 52134 & 8717 & 4737 & 3251 & 2475 & 1675 & 46668 & 6455 & 2451 & 42669 \\
\hline
\end{tabular}

Table 2: Coefficients $C_{2 k+1,2 j+1}, B_{2 k+1}$, and $A_{0}$ for various values $\eta$.

The amplitude of deflection is proportional to $A_{0}$; it essentially depends on the ratio $\eta$ (see Table 2), but the deflection mode depends slightly on $\eta$ and is close to the function $\sin \left(\pi x_{1} / a_{1}\right)$ (see Fig. 2).

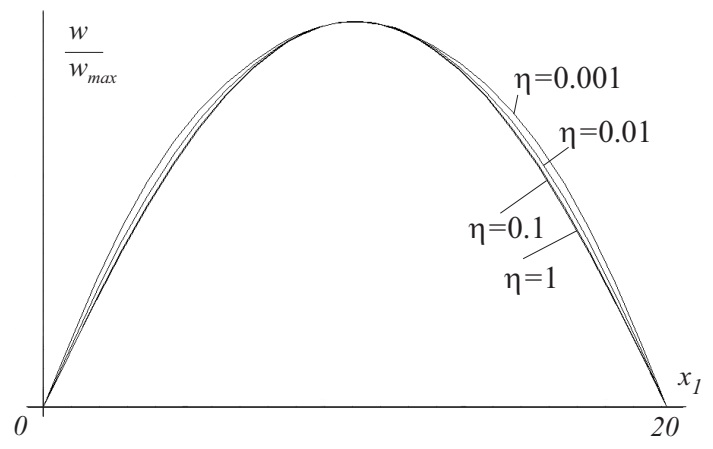

Figure 1: Deflection modes for various values of the ratio $\eta$ of the Young moduli. 


\section{OPTIMAL DISTRIBUTION OF THE LAYER THICKNESSES}

Consider a multilayer plate with the alternating hard and soft layers. Let the entire number of layers be $n=2 n_{0}+1$ with $n_{0}+1$ layers and $n_{0}$ soft layers. We set

$$
h_{h}=\sum_{k=0}^{n_{0}} h_{2 k+1}, \quad h_{s}=\sum_{k=1}^{n_{0}} h_{2 k}, \quad h=h_{h}+h_{s},
$$

where $h_{h}$ and $h_{s}$ are the summary thicknesses of the hard and of the soft layers, respectively. Assume that the values $h_{h}, h_{s}$, also the elastic moduli of hard and of soft layers, and the thickness parameter $\mu$ are given. We seek the distribution of the layer thicknesses $h_{k}$ satisfying Eqs. (39), for which the plate bending stiffness is maximum. As the bending stiffness we accept the inverse value the maximum deflection $f\left(h_{1}, h_{2}, \ldots, h_{n}\right)=w(0)^{S A}$ (see Eq. (30)).

In most cases a three-layer plate with

$$
n=3, \quad h_{1}=h_{3}=h_{h} / 2, \quad h_{2}=h_{s}
$$

has the maximum stiffness. But if the ratio $\eta$ of elastic moduli is very small, then the other distribution of thicknesses may lead to the maximum stiffness. Here we investigate this problem in details for the fixed values $\mu=0.3776, \nu_{1}=\nu_{2}=0.3$, for two changing parameters $\eta$ and $s=h_{h} / h$, and for $n \leq 11$ (without loss of generality we may take $E_{1}=1$ and $h=1$ ). The small parameter $\mu=0.3776$ corresponds, in partial, to a rectangular plate with $a_{1}=10, a_{2}=15$.

To find the thickness distribution corresponding to the maximum stiffness for the fixed values $\eta$ and $s$, we seek the minimum of function $f\left(h_{k}\right)=f\left(h_{1}, h_{2}, \ldots, h_{n}\right)$ in $h_{k}$ satisfying Eqs. (39). To find $h_{k}^{0}$ that delivers the minimum to the function $f\left(h_{k}\right)$ we use the Monte Carlo method in combination with the method of iterations [18]. Thanks to Eqs. (39) the point $h_{k}$ moves in the space $R^{n-2}$. Let $h_{k}^{(i)}$ be some point. We next seek the next point $h_{k}^{(i+1)}$ for which

$$
f\left(h_{k}^{(i+1)}\right)<f\left(h_{k}^{(i)}\right)
$$

as follows. We put $h_{k}^{*}=\max \left\{0, h_{k}^{(i)}+\xi_{k}\right\}$, where $\xi_{k}$ are the uniformly distributed numbers in $[-\varepsilon, \varepsilon]$ (for example, $\varepsilon=0.01$ ). Then we normalize the values $h_{k}^{*}$ according to Eqs. (39)

$$
h_{2 i+1}^{* *}=\frac{s h_{2 i+1}^{*}}{\sum_{i=0}^{5} h_{2 i+1}^{*}}, \quad h_{2 i}^{* *}=\frac{(1-s) h_{2 i}^{*}}{\sum_{i=1}^{5} h_{2 i}^{*}}
$$

If $f\left(h_{k}^{* *}\right)<f\left(h_{k}^{(i)}\right)$, then we take $h_{k}^{(i+1)}=h_{k}^{* *}$. Otherwise, we repeat the previous step with different random numbers $\xi_{k}$.

As the initial distribution we take $h_{1}^{(1)}=h_{11}^{(1)}=s / 2, h_{6}^{(1)}=(1-s)$, assuming that the rest of $h_{k}^{(1)}$ is 0 . Calculations show that it is enough to make $10^{6}$ steps.

The curve tn Fig. 3 divides the plane $(s, \eta)$ of the problem parameters into two parts; in one of which the three-layer plate has maximum stiffness.

Consider in more detail the case $s=1 / 3, \mu=0.3776$ with various values of the parameter $\eta$. For $\eta>\eta_{*}=0.0122$ the three-layered plate (40) has maximal bending stiffness. In Table 3 for various $\eta$ the minimal deflection, $W_{o}$, as found by the algorithm described above, is compared with the deflection, $W_{u}$, of a plate with the uniform thickness distribution (see Section 4) and with the deflection, $W_{3}$, of the three-layered plate (40). For $\eta>\eta_{*}$ the three-layered plate is optimal, $W_{3}=W_{o}$. For $\eta<0.006$ the stiffness of a plate with uniform thickness distribution is larger than the stiffness of a three-layer plate, $W_{u}<W_{3}$. 


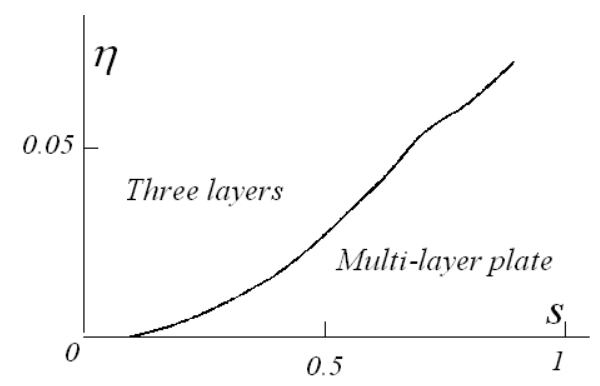

Figure 2: The plane $(s, \eta)$ for $\mu=0.3776$.

\begin{tabular}{|l|r|r|r|}
\hline$\eta$ & uniform, $W_{u}$ & three-layered, $W_{3}$ & optimal, $W_{o}$ \\
\hline 0.001 & 15617 & 17812 & 11539 \\
0.002 & 8446 & 9286 & 7815 \\
0.005 & 4139 & 4170 & 4007 \\
0.01 & 2697 & 2464 & 2458 \\
0.0122 & 2434 & 2155 & 2155 \\
0.02 & 1962 & 1607 & 1607 \\
0.1 & 1268 & 901 & 901 \\
0.5 & 785 & 663 & 663 \\
\hline
\end{tabular}

Table 3: Comparison of the stiffness parameters for various multilayer plates with $\mu=0.3776, s=1 / 3$.

In Table 4 the optimal distribution of thicknesses for $s=1 / 3, \mu=0.3776$, and for four values of $\eta$ is presented. The thicknesses of soft layers are shown in italic. For $\eta=0.01$ the distribution is symmetric with respect to the mid-plane. With smaller $\eta$ the distribution is not symmetric. The resulting asymmetry can be accounted for by the fact that the value $W_{o}$ is the deflection of the plane $z=0$ to which the external force is applied. At $\eta=0.001$ the optimal plate degenerates to a three-layer plate with very dissimilar distribution of layers.

\begin{tabular}{|l|ccccccccccc|}
\hline$\eta$ & $h_{1}$ & $h_{2}$ & $h_{3}$ & $h_{4}$ & $h_{5}$ & $h_{6}$ & $h_{7}$ & $h_{8}$ & $h_{9}$ & $h_{10}$ & $h_{11}$ \\
\hline 0.01 & 0.149 & 0.192 & 0.007 & 0.099 & 0.010 & 0.081 & 0.010 & 0.099 & 0.007 & 0.192 & 0.149 \\
0.005 & 0.094 & 0.212 & 0.025 & 0.090 & 0.048 & 0.063 & 0.047 & 0.090 & 0.025 & 0.211 & 0.095 \\
0.002 & 0.030 & 0.255 & 0.024 & 0.075 & 0.213 & 0.039 & 0.027 & 0.083 & 0.011 & 0.215 & 0.029 \\
0.001 & 0.331 & 0.667 & 0.002 & - & - & - & - & - & - & - & - \\
\hline
\end{tabular}

Table 4: Distribution of thicknesses of the optimal plate with $s=1 / 3, \mu=0.3776$.

The similar results also hold for a rectangular plate with the Navier boundary conditions, because the first summand in the expression for $w\left(x_{1}, a_{2} / 2,0\right)$ in Eq. (38) is much larger than the rest summands.

The results of Section 6 are approximate, since they are based on the approximate equation (30). The error in Eq. (30) is estimated in Section 4 (see Table 1). The exact results may be obtained by numerical solution of Eqs. (9). But for the large number of iterations this approach takes a long time, though the result is almost the same. 


\section{CONCLUSIONS}

- A two-dimensional linear model of second-order accuracy (SA) is used for analysis of a thin multi-layer plate bending.

- The formula for an infinite plate deflection subject to a doubly periodic external force is obtained. This formula is applied to a rectangular multi-layer plate with alternating hard and soft layers and with the Navier boundary conditions.

- The plate deflection depends on the distribution of thicknesses of hard and soft layers.

- The problem of the optimal distribution, which gives the maximum bending stiffness, is attacked using the Monte Carlo method.

- For a very small ratio $\eta$ of the elastic moduli the three-layer plate with the soft layer lying between two hard layers was found not to be optimal. In this case, the plate with the maximum bending stiffness is the multi-layered one with the number of layers $n>3$.

- The plate with $n=11$ layers is examined.

\section{ACKNOWLEDGEMENTS}

This research was carried out with the financial support of the Russian Foundation for Basic Research (grants no. 16-01-00580a, 14-01-00271a, 16-51-52025 MHTa).

\section{REFERENCES}

[1] G. Kirchhoff, Vorlesungen uber Matematische Physik. Mechanik, Leipzig, 1876 [in German].

[2] A.E.H. Love, A treatise on the mathematical theory of elasticity, Cambridge Univ. Press, 1927.

[3] S.P. Timoshenko, On the correction for shear of the differential equation for transverse vibrations of prismatic bars, Philos. Mag., 4, Ser. 6, no. 242, 1921.

[4] E. Reissner, The effect of transverse shear deformation on the bending of elastic plates, Trans. ASME, J. Appl. Mech., 12, 69-77, 1945.

[5] K.F. Chernykh, V.A. Rodionova, B.F. Titaev, Applied theory of anisotropic plates and shells, St.Petersburg Univ. Press, 1996 [in Russian].

[6] I.N. Vekua, On one method of calculating prismatic shells, Trudy Tbilis. Mat. Inst., 21, 191-259, 1955 [in Russian].

[7] A.L. Goldenweizer, Theory of Elastic Thin Shells, Pergamon Press, London, 1961.

[8] P.E. Tovstik, T.P. Tovstik, A thin-plate bending equation of second-order accuracy, Doklady Physics, 59, no. 8, 389-392, 2014.

[9] Y. Vetyukov, A. Kuzin, M. Krommer, Asymptotic splitting in the three-dimensional problem of elasticity for non-homogeneous piezoelectric plates, Int. J. Solids and Structures, 48, 12-23, 2011. 
[10] V.A. Eremeev, L.M. Zubov, Mechanics of elastic shells, Moscow, Nauka, 2008, [in Russian].

[11] H. Altenbach, G.I. Mikhasev (eds.), Shell and membrane theories in mechanics and biology, Springer (2014).

[12] J.N. Reddy, A refined nonlinear theory of plates with transverse shear deformation, Int. J. Solids and Structures, 20, 881-896, 1994.

[13] S.P. Timoshenko, Strength of materials, D. Van Nostrand, Toronto, New York, London, 1956.

[14] V. Birman, Plate Structures, Springer, Dordrecht, 2011.

[15] N.F. Morozov, P.E. Tovstik, Bulk and surface stability loss of materials. In: Multi-Scaling of Synthetic and Natural Systems with Self-Adaptive Capacity, Taiwan, 27-30, 2010.

[16] R. Kienzler, P. Schneider, Comparison of various linear plate theories in the light of a consistent second order approximation, Shell Structures: Theory and Applications. Proc. 10th SSTA 2013 Conf., 3, 109-112, 2014.

[17] P.E. Tovstik, T.P. Tovstik, On the 2D models of plates and shells including shear, ZAMM, 87, no. 2, 160-171, 2007.

[18] G. Winkler, Image analysis, random fields and dynamic Monte Carlo methods, SpringerVerlag, Berlin, 1995.

[19] O.C. Zienkiewicz, R.C. Taylor, The finite element method, Vol. I, 4th Edition. McGraw Hill, 1989.

[20] J.T. Oden, T. Belytschko, I. Babuska, T.J.R. Hughes, Research directions in computational mechanics. Computer Methods in Applied Mechanics and Engineering, 192, 913-922, 2003.

[21] J.H. Argyris, M. Papadrakakis, L. Karapitta, Elastoplastic analysis of shells with the triangular element TRIC. M. Papadrakakis, A. Samartin, E. Oñate eds. 4th International Colloquium on Computation of Shell and Spatial Structures (IASS-IACM 2000), Chania, Crete, Greece, June 4-7, 2000. 